\title{
La transmission des savoir-faire : Un objet pour l'ethnologie des techniques?
}

The Transmission of know-how: an object for the ethnology of technics?

\section{Marie-Noëlle Chamoux}

\section{(2) OpenEdition}

1 Journals

Édition électronique

URL : https://journals.openedition.org/tc/4995

DOI : $10.4000 /$ tc. 4995

ISSN : 1952-420X

Éditeur

Éditions de l'EHESS

\section{Édition imprimée}

Date de publication : 30 juin 2010

Pagination : 139-161

ISSN : 0248-6016

\section{Référence électronique}

Marie-Noëlle Chamoux, «La transmission des savoir-faire : Un objet pour l'ethnologie des

techniques? », Techniques \& Culture [En ligne], 54-55 | 2010, mis en ligne le 30 janvier 2013, consulté le 29 septembre 2022. URL : http://journals.openedition.org/tc/4995; DOI : https://doi.org/10.4000/tc. 4995 


\section{LA TRANSMISSION DES SAVOIR-FAIRE}

\section{Un objet pour l'ethnologie des techniques?}

in Techniques et culture, Bulletin de l'Équipe de recherche 191 (3), 1978 : 46-83

Pour analyser les relations entre techniques et culture, le choix d'un ordre pratique d'étude se pose en permanence. Par où commencer? Doit-on d'abord examiner en détail les rapports entre la matière et les hommes (recueil et traitement des données sur les outils, les matériaux, les procédés, etc.) et seulement après leur explicitation entreprendre l'analyse des rapports sociaux? Ou bien doit-on adopter l'ordre inverse et commencer par les rapports sociaux? S'il ne s'agissait que d'un ordre pratique, la question pourrait paraître bien secondaire. Mais la difficulté provient de ce que l'ordre pratique tend à imposer un ordre hiérarchique dans l'explication: une fois répertoriés et classés les procédés techniques, par exemple selon des critères de cohérence interne, est-il encore possible de déceler l'action des rapports sociaux sur les procédés? N'est-on pas enfermé dans un déterminisme à sens unique, celui qu'exerceraient les lois de la matière sur les différentes instances de la réalité sociale?

À la suite de Leroi-Gourhan, l'ethnologie spécialisée dans les techniques a le plus souvent donné la priorité à l'étude des rapports à la matière. Elle a recherché des classifications des outils et des procédés. Elle a aussi tenté d'en faire le répertoire le plus complet possible. Mais si cette démarche a permis de fructueuses comparaisons entre les techniques de divers lieux et de diverses époques, renforçant ou corrigeant les grandes hypothèses sur l'Évolution et les relations interculturelles, elle suffit rarement à saisir dans toutes leurs dimensions les liens entre les techniques d'un groupe particulier et les autres instances de sa réalité sociale.

Parallèlement à cette démarche classificatrice, on dispose dans l'ethnologie de très nombreuses descriptions comportant des informations sur les outils et les procédés, mais 


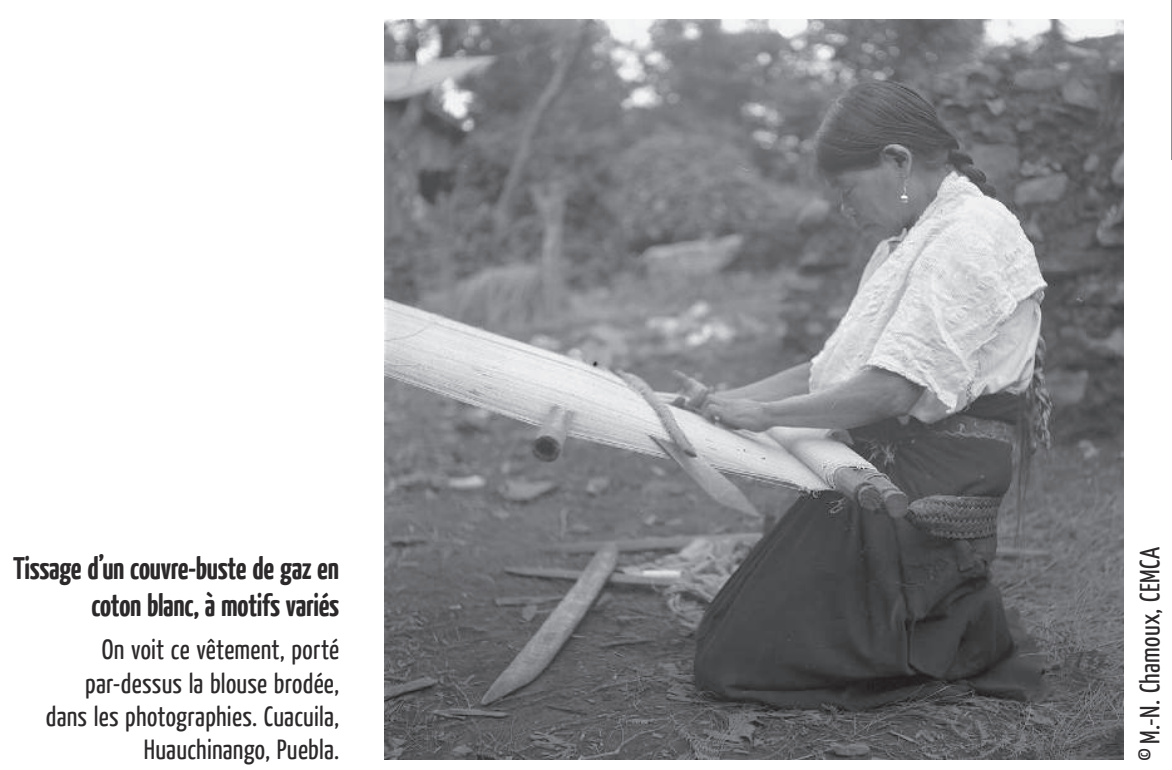

aussi sur d'autres dimensions des techniques: symbolisme, langages spécialisés, fonctions sociales des hommes qui les pratiquent, etc. On pouvait espérer qu'en rassemblant ces données éparses certains des liens entre techniques et culture s'expliciteraient d'eux-mêmes. Malheureusement rares sont les descriptions de techniques qui sont vraiment utilisables, lorsqu'on veut comparer, sans tomber dans un déterminisme sommaire, les rapports à la matière et les rapports sociaux. Les descriptions donnent l'impression d'être incomplètes, a-systématiques; les éléments semblent avoir subi une sélection arbitraire. Finalement les lacunes les plus courantes se situeraient moins dans la description des rapports sociaux que dans le recueil de données pertinentes sur les rapports à la matière ${ }^{1}$.

D’un côté, on a une systématique des rapports outils, procédés et matière, mais sans possibilité d'inclure les dimensions sociales des techniques dans la systématique; de l'autre côté, on trouve des pratiques empiristes de recueil, ne donnant que des aperçus limités de liens possibles entre techniques et culture. Que faire devant cette situation? On peut certes perfectionner les méthodes de descriptions des techniques pour tenter d'y inclure toutes les dimensions matérielles, sociales, culturelles qu'elles mettent en ouvre. C'est même une tâche nécessaire. Mais on peut douter qu'une telle entreprise ait jamais une fin. Aussi plutôt que de la poser comme un préalable à la réflexion sur les rapports entre techniques et culture, on proposera ici une autre modalité d'approche, non exclusive de la précédente. La tentative consiste à privilégier et systématiser un objet d'étude précis, qui intègre d'emblée des dimensions « purement » techniques et des dimensions socioculturelles: les savoir-faire techniques.

Par savoir-faire techniques, on entend l'ensemble des connaissances et savoirs humains, conscients ou inconscients, qui permettent la mise en œuvre d'une technique. Les savoirfaire peuvent être gestuels et intellectuels, collectifs et individuels, et ils dépendent toujours à la fois des rapports des hommes entre eux et des rapports entre les hommes et les lois de la matière.

L'étude des savoir-faire techniques est particulièrement cruciale dans l'étude des techniques non industrielles, et c'est bien ce qui fait toute la difficulté de l'ethnologie des techniques. On entend parfois dire que les techniques non industrielles seraient «plus simples ». Si l'on entend par là que les moyens de travail (outils) sont plus simples, la proposition est évidente. On peut en effet reconnaître que l'évolution des moyens de travail s'est faite grosso modo du plus simple au plus complexe, au plus élaboré. Mais si l'on 
entend par là que les tâches humaines individuelles sont plus simples dans les techniques anciennes on cède au contraire à un préjugé classique erroné. La sociologie du travail, qui d'ailleurs ne s'est intéressée qu'au travail industriel, a établi en effet que, contrairement à ce que l'on croit parfois, le progrès technique moderne a consisté à simplifier ou appauvrir toujours plus les tâches des exécutants, entraînant ce que l'on nomme une « déqualification» des travailleurs directs.

Selon qu'on se place dans le cas de techniques à base d'outils ou de techniques à base de machines, on se trouvera devant l'une ou l'autre des relations suivantes:

1. Outils « simple »/ Tâche individuelle « riche»

2. Machine «élaborée »/ Tâche individuelle « appauvrie».

En d'autres termes, les techniques non industrielles ne sont pas plus simples. Ce sont les outils qui le sont. Le travail individuel est au contraire plus élaboré : la manière de se servir de l'outil est plus importante que l'outil lui-même. On exprimait cela autrefois dans les leçons de morale de l'École Communale en enseignant que « les mauvais ouvriers ont toujours de mauvais outils ». Il ne suffit pas de posséder un métier à tisser amérindien, ni même d'avoir vu une Indienne s'en servir, pour pouvoir produire la même étoffe qu'elle. La manière d'utiliser un outil s'incarne concrètement dans les connaissances techniques conscientes ou non du travailleur, et dans les gestes qu'il effectue au cours du procès de travail. En un mot dans les savoir-faire techniques.

Dans les techniques industrielles, une partie du savoir-faire technique du travailleur humain lui a été ôtée et a été en quelque sorte transférée dans la machine. Il n'est besoin par exemple d'aucune connaissance spéciale, hormis une très courte période de formation, pour produire sur un métier à tisser de l'industrie un tissu identique, quelle que soit la personne surveillant le métier. La part d'habileté personnelle du travailleur dans l'obtention du résultat est moindre dans les techniques industrielles.

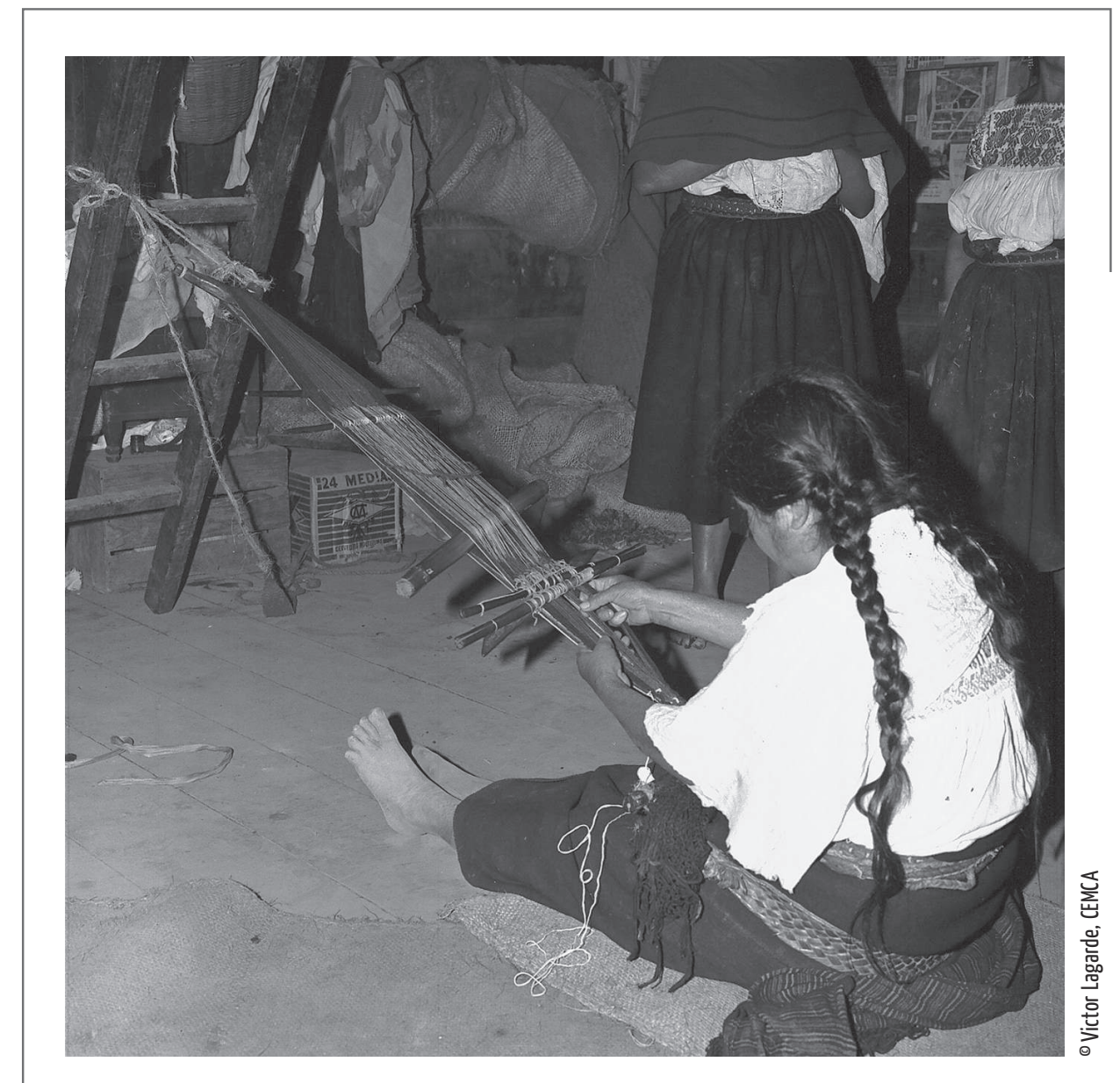

\section{Tissage d'une ceinture de broché en coton blanc} et laine rouge et verte, à motifs variés

On voit le port de ces ceintures dans les photographies. Cuacuila, Huauchinango, Puebla. 
Privilégier l'étude des savoir-faire techniques se justifie donc par la place qu'ils tiennent dans les techniques non industrielles. Mais c'est aussi choisir la part la plus difficilement cernable, la plus fuyante, la plus cachée à l'observateur et parfois au praticien lui-même. Yves Barel, reconnaissant le problème, propose une terminologie qui pourrait être utile pour formuler les questions posées ici. Il distingue les «savoir-faire incorporés » et les « savoir-faire algorithmisés ».

« Le travail est d'abord une activité qui repose soit sur un savoir-faire incorporé, soit sur la maitrise d'algorithmes. Un savoir-faire incorporé est un savoir-faire indissociable d'individus ou de groupes concrets: il est le résultat de leur apprentissage personnel, de leur expérience, de leur habileté. La caractéristique la plus importante du savoir-faire incorporé est qu'il n’est pas analysable et décomposable jusqu'au bout (le cas où l'analyse et la décomposition totales sont possibles, mais non tentées, est un cas limite). Le travailleur sait faire, mais il ne sait pas complètement comment il sait. Le savoir-faire incorporé n'est donc pas transmissible par enseignement. Il n'est transmissible que par apprentissage c'est-à-dire par la reproduction plus ou moins à l'identique d'individus ou groupes au cours du travail lui-même. Le support du savoir-faire est humain et biologique. Mais quand le savoir-faire est analysable et décomposable jusqu'au bout le savoir et le faire peuvent se déconnecter. Le savoir s'incorpore alors dans un rapport non humain: un livre, un traité, un programme, une fiche d'instruc-

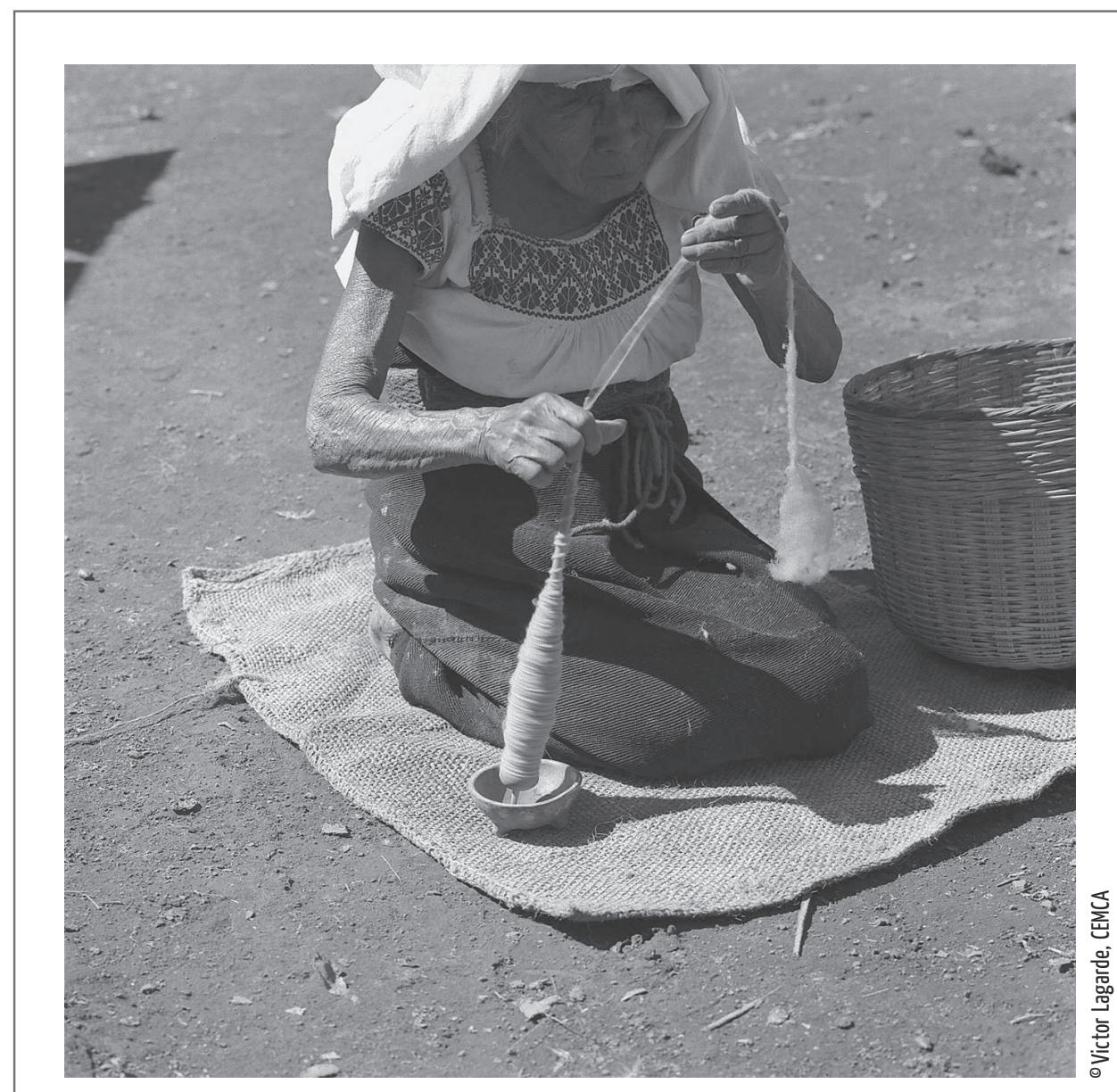

une impossibilité de pousser jusqu'à son terme cette algorithmisation: de nouveaux savoir-faire incorporés naissent de la dynamique même de l'algorithmisation. $»^{2}$

Pour ne pas alourdir l'exposé, on ne s'attardera pas ici à la dialectique évoquée par Barel entre les savoir-faire incorporés et les savoir-faire algorithmisés au cours de l'Histoire. Il faudrait pour cela discuter de la genèse de la société industrielle et de l'extension de ses pratiques spécifiques en matière de travail ainsi que de la prétention moderne de rationalisation totale du travail (OST) $)^{3}$.

Il est hors de doute que, dans de nombreuses populations étudiées par les ethnologues, les savoir-faire incorporés dominent largement. En un sens, la tâche scientifique de l'ethnologie des techniques pourrait être d'algorithmiser les savoir-faire incorporés. Même si, comme on peut 
l'admettre avec Barel, l'algorithmisation ne peut jamais être totale, s'il y a toujours une part de l'activité technique qui échappe à la mise en formule et à la programmation, même dans les formes les plus « scientifisées » des techniques (techniques industrielles), ne serait-il pas plus scientifique de prendre les savoir-faire incorporés tels quels et reconnaître d'emblée leur propriété d'échapper à l'algorithmisation totale?

Pourtant, quelle que soit l'impossibilité de formulation complète des savoir-faire, les groupes humains parviennent bien à les transmettre malgré tout. Pourquoi ne pas chercher alors à élucider ce processus lui-même? Pourquoi ne pas prendre pour objet les variations pertinentes des manières de transmettre les savoir-faire incorporés? C'est ce qui va être tenté. Pour illustrer ces processus, on commencera par évoquer un exemple banal de transmission de savoir-faire, en préalable à une grille analytique de la répartition dans le groupe et des modes de transmission des savoir-faire incorporés.

On analysera le cas d'Indiens nahuas du Mexique oriental, d'après des données recueillies directement sur le terrain.

\section{Un exemple d'apprentissage à la manière indigène}

L'ethnographie pourrait se définir comme une transmission partielle à l'ethnologie des savoir-faire de la population étudiée. Aussi les péripéties d’une enquête de terrain sont-elles presque toujours riches d'informations sur les méthodes pédagogiques en vigueur dans le groupe. Une expérience vécue assez banale peut l'illustrer, bien qu'elle ne concerne pas les techniques de production, mais les rituels.

Entre 1969 et 1971, j’ai séjourné dans le village nahua de Cuacuila, au nord de l'État de Puebla. Le village est réputé dans toute la région pour ses chamans (tlamatque). Une vieille Indienne, ne parlant que le nahuatl, était connue comme tlamatque mais surtout comme étant la meilleure marieuse. Les Nahuas emploient en effet un (ou une) intermédiaire connaissant les rites pour négocier une alliance, selon une coutume d'origine pré-colombienne. J'allai voir cette femme pour lui demander quelques explications sur les rites actuels du mariage. C'était la démarche classique en sciences sociales de l'entretien sur un thème.

Or rien n'était plus étranger à l'expérience de la vieille Antona'tzin que de parler sur un thème: toute sa vie, elle n'avait parlé que pour obtenir un résultat. Elle traduisit donc ma demande dans un sens, pour elle, intelligible: elle comprit que je voulais apprendre moi-même les rites pour pratiquer la spécialité de marieuse et elle m’opposa un «non! » très ferme. Je n'insistai pas. Quelques semaines plus tard, elle se ravisa et vint me dire que je me tienne prête pour le lendemain matin à l'aube. À partir de ce jour, elle m'emmena avec elle dans les fiançailles et les mariages où elle devait officier. Bien plus, elle me fit assister, sans que je lui aie demandé, à certains rites du culte des Pluies et des Vents qu'elle pratiquait. J'étais donc aux premières loges pour observer comment elle s'y prenait pour transmettre un savoir-faire rituel.

Sa méthode consistait à me faire assister, à ses côtés, au déroulement entier du rituel, y compris des phases qui ne sont pas habituellement effectuées en public. Je n'ai pratiquement jamais reçu d'explications verbales sur la conception du monde et la symbolique qui sous-tend ces rites nahuas. Tout au plus, parfois, dans une circonstance ordinaire de la vie quotidienne, elle donnait une bribe d'information. 
On pourrait supposer que cette femme manquait de moyens linguistiques. Rien n'est plus faux: les fonctions de chaman et de marieuse reposent toutes deux sur la maîtrise de prières et d'incantations dans un langage riche en termes rares, en métaphores, et en tournures grammaticales marquant le respect qu'on n'emploie que dans les rites. De plus, ces prières et incantations n'ont pas le caractère de formules préfabriquées, d'algorithmes qu'il suffirait d'apprendre par cour pour devenir chaman. Chaque tlamatque nahua les construit pour lui-même, en puisant bien sûr les éléments dans le fonds commun culturel de son groupe. Son savoir spécifique n'est pas un savoir tout court, mais un savoir-dire et un savoir-faire cérémoniel. La parole rituelle est, pour les Nahuas, habileté personnelle, et non répétition de formules plus ou moins secrètes. On est à l'opposé de la conception catholique de la prière et du rite, et des traditions de la sorcellerie européenne. Cette femme non seulement répétait les mots du langage sacré, mais elle était capable de produire ce langage, comme tous les tlamatque. Elle avait à sa disposition davantage de moyens linguistiques que les gens ordinaires.

Il existe des travaux ethnographiques reproduisant des explications détaillées données par les chamans eux-mêmes sur la conception du monde et le symbolisme indigène. J'ai moi-même recueilli de telles explications, auprès d'autres Nahuas. Si Antona'tzin ne m'expliquait presque rien, c'est que j'étais pour elle une élève à qui elle essayait de transmettre un savoir-faire, et non pas sa théorie religieuse personnelle. Ce qui importait, c'était de me montrer comment on fait; le reste viendrait de surcroît. Les tlamatque nahuas n'ont pas les ambitions philosophiques des gourous, et ne les auront pas tant que des agences de tourisme ne créeront pas un marché d'exotisme chamaniste.

Cette pédagogie du montrer comment on fait est appliquée à diverses activités, à Cuacuila. Sans exclure par avance l'existence d'autres méthodes encore à découvrir, on peut dire qu'elle est dominante dans l'agriculture, le petit commerce, les tâches domestiques masculines et féminines.

\section{Esquisse d'une grille analytique des savoir-faire incorporés}

Prendre pour objet des savoir-faire incorporés, non algorithmisés, exige de dépasser une confusion trop souvent pratiquée au niveau même du recueil des données. Les descriptions techniques aboutissent fréquemment à la rédaction de modes d'emploi, de recettes techniques. Bref, elles tentent d'établir elles-mêmes une algorithmisation, absente dans les sociétés étudiées. Mais de tels modes d'emploi sont insuffisants pour retracer véritablement les liens entre technique et société. Cela tient à ce que les descriptions de ce type sont implicitement normatives (ce qu'il faut faire pour obtenir tel ou tel produit). Elles laissent alors de côté tout un ensemble de phénomènes relatifs aux conditions concrètes de la mise en œuvre des techniques. Autrement dit, elles ignorent, pour un même procédé, les variations possibles de la manière d'agir. Elles ignorent l'ensemble des conditions sociales qui permettent la réalisation et la reproduction des savoir-faire. Pour marquer la dualité entre le plan des modes d'emploi et le plan de la mise en œuvre concrète, on parlera, dans le second cas, de pratiques techniques.

La notion de pratiques techniques offre un double intérêt. En premier lieu, mieux que le mot « techniques », elle permet de souligner la dualité entre d'une part les procès techniques concrets et d'autre part leur mise en algorithme par l'ethnologue ou éventuellement 
par le groupe indigène, qu'on peut appeler aussi discours technique. En second lieu, la notion invite à mettre en évidence les décalages entre la réalité technique et les discours sur la technique, c'est-à-dire les constructions indigènes idéologiques et symboliques qui s'y rapportent.

Les pratiques techniques peuvent varier d'une population à l'autre, même si les procédés connus sont identiques. Pour étudier les pratiques techniques d'un groupe donné, par exemple d'un village, le moyen est de considérer les variations des savoir-faire techniques selon leurs différents supports humains individuels ou collectifs. Quelques-unes de ces variations pouvant avoir une portée générale vont être examinées.

\section{Quelques dimensions des savoir-faire}

Une première variation semble très répandue: les savoirs techniques et les activités techniques réelles des individus ne se confondent pas entièrement. C'est une chose que de posséder une technique, et une autre chose que de la pratiquer effectivement. En transposant des termes utilisés en linguistique, on dirait qu'il existe une compétence technique et des performances techniques. Dans les savoir-faire incorporés, il semble qu'il puisse exister une compétence sans performance (on sait faire, mais on ne fait pas) et une compétence avec performance (on sait faire et on fait) ${ }^{4}$.

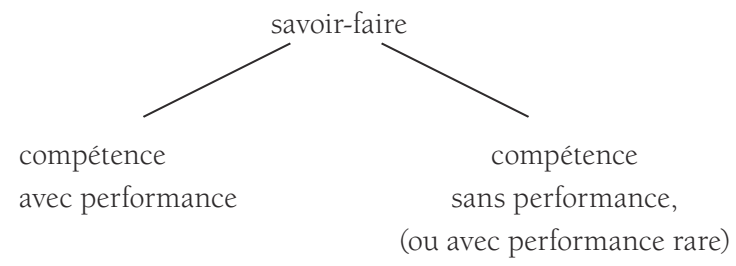

Cette distinction a le mérite d'être très simple. Malgré cela elle est rarement faite, et bien souvent, en ethnologie, la performance technique est considérée comme le signe co-extensif de la compétence, ce qui sous-entend que l'absence du « faire » est signe d'absence du « savoir-faire ». Or il n'en est pas nécessairement ainsi. On le verra plus loin en étudiant un exemple.

Auparavant, on considérera une autre variation, elle aussi très générale, dans les savoir-faire: les différences d'extension dans le groupe ou le village. Tous les savoir-faire incorporés ne sont pas transmis à tout le monde. Certains sont bien communiqués à tous: on les appellera savoir-faire généraux. D’autres sont transmis seulement à certaines personnes: les savoir-faire particuliers. Les différences d'extension des savoir-faire délimitent des sous-groupes dans la population considérée.

Dans une population réelle, certains sous-groupes pré-existent à ceux qui sont découpés par les variations de savoir-faire. Il s'agit en particulier de la distinction entre hommes et femmes ${ }^{5}$. Pour ne pas opposer quelques savoir-faire généraux à toutes les sortes de savoir-faire particuliers, on conviendra que des savoir-faire distincts correspondant aux deux groupes de sexe seront considérés comme généraux: on parlera de savoir-faire généraux masculins et de savoir-faire généraux féminins. Le principal avantage de cette convention est de réserver l'expression de savoir-faire particuliers aux différents cas d'activités de 
spécialistes individuels ou collectifs. La combinaison des deux types de savoir-faire et des groupes humains retenus donne six cas possibles:

- Savoir-faire généraux

- Savoir-faire généraux masculins

- Savoir-faire généraux féminins

- Savoir-faire particuliers

- Savoir-faire particuliers masculins

- Savoir-faire particuliers féminins

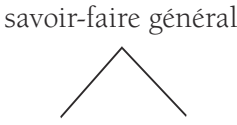

masculin féminin $\backslash$ pour tous $/$ savoir-faire particulier

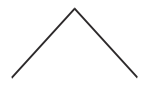

masculin féminin

pour tous $/$

En conjuguant les deux variations des savoir-faire permettant de caractériser les pratiques techniques, c'est-à-dire les variations de compétences et les variations d'extension dans le groupe, on obtient le diagramme suivant.

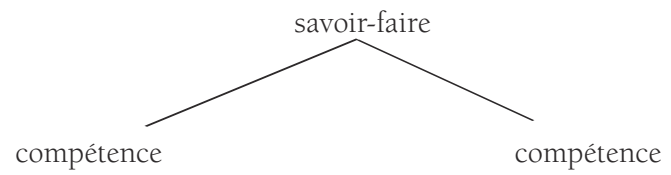

avec performance
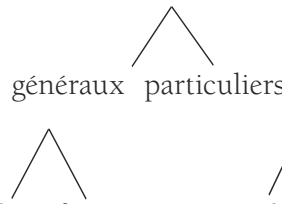

masculins féminins masculins féminins

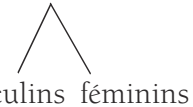

masculins (ou avec performance rare)
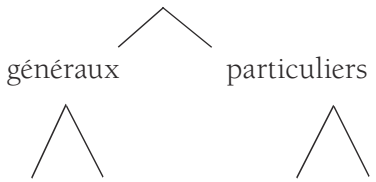

féminins masculins féminins

L'étude de ces variations de pratiques techniques est une manière d'aborder le thème de la division du travail, central dans l'étude des techniques. L'application de la grille de décryptage des savoir-faire peut donner des résultats différents de ceux qu'on obtient habituellement lorsqu'on considère la répartition des « activités » ou « tâches » selon les sexes et les spécialités, c'est-à-dire lorsqu'on ne retient que les performances techniques. On va en faire l'expérience à partir de données recueillies chez les Nahuas de Cuacuila.

\section{Un exemple d’application : la division sexuelle du travail chez les Nahuas}

L'ethnographie du village étudié fournit certaines données en matière de division du travail. Pour les Nahuas de Cuacuila, l'agriculture est l'activité masculine par excellence. Des rites le soulignent symboliquement: dans le cercueil d'un homme mort on dépose 
des instruments aratoires en miniature. Au cours de la vie quotidienne, ce sont effectivement les hommes qui pratiquent l'agriculture, qui décident des opérations à effectuer et des moments où les faire. Les femmes n'interviennent comme travailleuses que dans trois opérations culturales: l'épandage de l'engrais, le repiquage des plants pour certaines cultures, et la récolte. Le labour, les semailles, le sarclage sont des travaux d'homme.

Cette répartition sexuelle du travail agricole est volontiers exprimée et justifiée par les hommes de Cuacuila: les femmes sont « trop faibles » pour sarcler. L'épandage de l'engrais, le repiquage et la récolte sont des travaux « faciles », qui ne demandent ni force ni habileté particulière. En les faisant, elles peuvent aider aux champs « selon leurs petits moyens ».

Les femmes nahuas reprennent ces arguments: nous sommes faibles, nous ne pouvons pas, nous ne savons pas faire les travaux des hommes. Ce sont des travaux pénibles. C'est parce qu'ils nous traitent bien que nos maris et nos pères ne nous font pas faire les travaux les plus durs.

À s'en tenir à ces données faciles à recueillir, il semble évident que l'activité agricole est un attribut masculin, qu'elle est incorporée au groupe des hommes. Le discours technique et la symbolique indigènes concordent pour affirmer que l'agriculture est liée au corps biologique masculin, et disjointe, sauf pour des tâches très précises, du corps biologique féminin. Les performances techniques aisément observables renforcent la crédibilité de cette idée.

Pourtant une enquête plus approfondie révèle que les choses ne sont pas si simples, et que les femmes, contrairement à ce que l'idéologie indigène affirme, détiennent une part de compétence technique agricole, au-delà des tâches qui leur sont habituellement confiées.

Dans les cas de nécessité, par exemple si une famille manque de main-d'œuvre et est trop pauvre pour engager un journalier agricole, on verra des femmes manier le plantoir pour ensemencer et la houe pour sarcler. Ou encore s'il n'y a pas d'homme dans la maisonnée, elles prendront la direction des opérations de culture et de leurs journaliers. Leur compétence agricole se révèle également par l'enquête: beaucoup de femmes sont aussi capables que les hommes de donner des détails pertinents sur les chaînes opératoires de l'agriculture, d'en expliquer les difficultés et d'en préciser les techniques.

Quant à l'argument reposant sur la faiblesse physique des femmes, il est évidemment «ad hoc » : aucun Nahua ne songe à trouver trop dur pour les femmes certains travaux ordinaires, par exemple celui de porter une charge de bois sur le dos en même temps qu'un enfant sur la poitrine et un panier plein à la main, et ce durant plusieurs kilomètres en terrain montagneux...

Ainsi la division du travail entre hommes et femmes pour l'agriculture n'est pas vraiment fondée sur une division des compétences, sur une différence réelle de savoir-faire. La différence est surtout affirmée par le système nahua de représentations, qui présente l'agriculture comme un savoir-faire général masculin. Contrairement à ce que l'on aurait pu croire sur la foi des seuls entretiens, l'agriculture peut être considérée comme un savoir-faire général pour tous à Cuacuila. Il en est de même dans les villages voisins visités.

Une analyse symétrique peut être faite pour certaines tâches d'apparence typiquement féminines. La fabrication des galettes de maïs (tlaxcalli, tortillas en espagnol), base de l'alimentation, est un travail féminin biquotidien. Jamais on ne verra un homme faire des tortillas. L'idée est même perçue comme cocasse. Tous les hommes affirment, en riant, qu'ils ne savent pas les faire. Là encore, il n'est pas sûr qu'il faille les croire sur parole. On m’a rapporté que dans des circonstances exceptionnelles (nécessité de camper dans un endroit désert, par exemple) l'homme nahua se débrouillait infiniment mieux 
pour faire des tortillas que la citadine mexicaine de classe moyenne qui les achète au magasin du coin habituellement. Ainsi les hommes sont en réalité compétents, mêmes s'ils évitent de le montrer.

Contrairement aux apparences, la division sexuelle du travail chez les Nahuas ne repose pas toujours sur une division des compétences. Elle n'est parfois qu'une division des tâches entre hommes et femmes aux savoir-faire communs.

Les décalages entre compétence avec ou sans performance et l'idéologie indigène ne se font pas toujours dans ce sens. L'exemple du tissage est significatif d'un autre décalage possible. À Cuacuila le tissage, activité féminine, est symboliquement opposé à l'agriculture masculine. À la place d'instruments aratoires, on met une miniature de métier à tisser dans le cercueil d'une femme. Sur le plan symbolique, le tissage apparaît comme un attribut féminin, une technique liée au corps des femmes. C'est une conception générale chez les Indiens de l'Orient mexicain, comme l'indiquent de très nombreux témoignages ethnographiques. L'idéologie indigène fait du tissage un savoir-faire général féminin, contrastant avec l'agriculture, savoir-faire général masculin.

Mais cette opposition symbolique ne correspond pas à une opposition homologue en termes de savoir-faire. À Cuacuila, le tissage est aujourd'hui un savoir-faire particulier féminin. Autrement dit il est une activité de spécialiste, exclusivement féminine. En résumé, si l'on compare le statut apparent et les pratiques de l'agriculture et du tissage, on a la situation suivante. Au plan symbolique, les deux techniques sont dans une position symétrique et inverse. Au plan des pratiques techniques, ni l'un ni l'autre ne sont dans ces mêmes positions. L'agriculture, savoir-faire général masculin dans les symboles, est savoir-faire de tous dans la réalité. Le tissage, savoir-faire général féminin dans l'idéologie, n'est qu'un savoir-faire particulier féminin.

\begin{tabular}{|c|c|c|}
\hline & AGRICULTURE & TISSAGE \\
\hline Plan des symboles & Savoir-faire général masculin & Savoir-faire général féminin \\
\hline Plan des pratiques & Savoir-faire général pour tous & Savoir-faire particulier féminin \\
\hline
\end{tabular}

Non seulement les pratiques techniques diffèrent des représentations indigènes, mais les situations respectives des deux techniques considérées changent suivant le plan où l'on se place. Dans les pratiques, l'agriculture n'est pas dans le même rapport avec le tissage que sur le plan symbolique. Il n'y a pas d'isomorphisme des places respectives de l'une et l'autre technique selon les plans. La construction idéologique indigène est démentie par la réalité pratique, et elle n'est pas à son image, pas même à son image inversée.

Ces décalages ont-ils leur origine dans des changements récents intervenus dans les pratiques techniques? Y avait-il dans la période antérieure une correspondance ou tout au moins une isomorphie entre l'idéologie indigène et les pratiques techniques? Pour le tissage, il semble bien qu'il y ait eu évolution: d'après l'enquête, le tissage était effectivement connu et pratiqué de la plupart des femmes nahuas. Si cela est vrai, son statut symbolique et sa place réelle étaient les mêmes: un savoir-faire général féminin. Par contre pour l'agriculture, on n'a recueilli aucune indication remettant en cause dans le passé sa situation actuelle de savoir-faire général: rien ne permet de penser que les hommes nahuas aient eu antérieurement le monopole des savoirfaire agricoles. Si cela se vérifiait, le statut symbolique de l'agriculture n'aurait jamais correspondu aux pratiques. 
On aboutit donc à une interprétation inverse de ce que l'ethnologue aurait pu supposer spontanément. L'idéologie indigène fait croire en une division symétrique des compétences suivant les sexes. Les performances observables contredisent rarement cette idéologie. L'ethnologue sera porté à en déduire qu'il existe bien dans la réalité une division sexuelle des savoir-faire dont la symbolique indigène serait le simple reflet. Or, après l'analyse, le schéma doit être complètement renversé: il n'existe une compétence exclusive que d'un côté, celui des femmes. Mais l'idéologie indigène ne pouvait l'admettre. En conséquence elle a fabriqué une homologie symbolique à la compétence féminine: elle a inventé la compétence masculine en matière agricole.

Outre ce qu'elles disent sur les rapports entre techniques et culture, l'intérêt de telles comparaisons est double. D'abord, elles permettent de formuler des hypothèses sur les capacités d'adaptation d'une communauté villageoise face à des changements externes éventuels. Supposons qu'une migration massive des hommes nahuas intervienne. Elle n’entraînerait pas nécessairement la disparition des savoir-faire agricoles dans le village. Par contre le tissage, de par sa situation actuelle de savoir-faire particulier féminin, semble être plus fragile face à des bouleversements économiques ou démographiques. En second lieu, ces comparaisons fournissent une base pour aborder sérieusement l'étude des rapports sociaux entre hommes et femmes, thème pour lequel on se contente bien souvent de reprendre en termes savants les préjugés « machistes » véhiculés par notre culture, ou au contraire des slogans féministes tout aussi ethnocentriques. On ne développera pas ici ces thèmes. Mais il a semblé utile de souligner au passage la fécondité de l'étude des techniques, au-delà de la simple technographie.

\section{Les modes de transmission des savoir-faire incorporés}

Le fait que la méthode d'apprentissage puisse être la même pour toutes sortes d'activités techniques peut sembler contradictoire avec le fait que certains savoir-faire sont particuliers et d'autres généraux. Aussi faut-il s'interroger sur les mécanismes qui produisent les différences d'extension dans le groupe villageois. Puisqu'ils ne résident pas dans une opposition entre transmission par apprentissage et transmission par algorithme, ces mécanismes ne peuvent se situer que dans les différences de relation entre villageois. Autrement dit dans des différences de réseaux de transmission, de canaux d'incorporation.

À Cuacuila, on trouve deux types de réseaux indigènes de transmission. Ils seront distingués selon qu'il s'établit ou non une relation spécifique de maître à apprenti. On parlera de transmission par imprégnation dans les cas où le maître, c'est la famille tout entière, voire le village tout entier, autrement dit quand ne s'établit pas une relation spécifique d'apprentissage. Dans le cas contraire, on trouvera une transmission par un maître (maître informel ou maître institué).

Ces réseaux ne sont pas les seuls possibles en théorie. On peut en imaginer bien d'autres, et en rencontrer des illustrations dans diverses sociétés humaines. Mais on s'en tiendra là dans cet article, réservant pour plus tard l'examen d'un autre réseau existant dans le village, celui de l'école primaire d'État, et la recherche d'autres réseaux théoriquement possibles.

En considérant les deux réseaux présents à Cuacuila, une hypothèse se présente immédiatement à l'esprit: y aurait-il toujours une transmission par imprégnation pour les savoir-faire 
particuliers, ou vice-versa? L'ethnographie du village montre qu'il n'y a pas correspondance nécessaire, et que les quatre combinaisons logiquement possibles entre les extensions des savoir-faire et les réseaux de transmission peuvent effectivement se rencontrer.

\begin{tabular}{|c|c|c|}
\hline & $\begin{array}{c}\text { Transmission par imprégnation } \\
\text { A }\end{array}$ & $\begin{array}{c}\text { Transmission par un maitre } \\
\text { B }\end{array}$ \\
\hline Savoir-faire généraux a & $\mathrm{aA}$ & $\mathrm{aB}$ \\
\hline Savoir-faire particuliers b & $\mathrm{bA}$ & $\mathrm{bB}$ \\
\hline
\end{tabular}

Pour ne mentionner que quelques techniques parmi toutes celles qui correspondent aux quatre combinaisons, on peut citer pour Cuacuila: dans le cas aA, l'agriculture, certaines tâches quotidiennes de la vie domestique, le portage des charges; en bA, on aura la charcuterie, la boucherie; la combinaison aB s'illustre bien par la broderie et bB par le tissage, la musique...

En examinant des illustrations des quatre cas, on s'attachera à décrire plus particulièrement les modalités de la transmission par imprégnation. En outre, pour montrer dans quelles conditions peut s'imposer une relation de maître à apprenti, on devra rentrer dans le détail technique avec la description de la broderie et du tissage.

\section{Comment s'effectue la transmission des savoir-faire par imprégnation}

La compétence technique et la performance ne se recouvrent pas toujours, on l'a vu. Il semble bien que certains aspects du mode de vie des Nahuas favorisent la généralisation de certaines compétences au-delà des performances techniques, que par la large place que tient la transmission des savoir-faire par imprégnation.

Lapprentissage par imprégnation suppose deux conditions. En premier lieu, il s'appuie sur un entrainement corporel et intellectuel commun à tous les membres du groupe villageois: gestes, postures, mode de perception de la matière, langage... Cet entraînement se rattache à ce que l'on appelle généralement la culture du groupe. En second lieu, il suppose la répétition de l'observation des différentes techniques et de l'expérimentation des gestes. Qu'une de ces deux conditions ne soit pas remplie, et l'imprégnation ne se fait plus toute seule. Il est besoin d'un maître pour que le savoir-faire soit transmis.

\section{Dans les savoir-faire généraux}

Pour prendre des exemples dans les savoir-faire généraux, on peut se demander comment le savoir-faire agricole est-il transmis concrètement? Comment la compétence pour de nombreuses activités domestiques est-elle largement répandue? Chez les Nahuas, tant aux champs qu'à la maison et dans ses abords, il n'y a point de cloisonnement rigide dans la vie quotidienne entre hommes et femmes, jeunes et vieux; point de lieux réservés ou interdits; point d'activités secrètes. Dans l'espace agricole et domestique, les enfants des deux sexes ont accès à l'observation de différents savoir-faire aussi longtemps qu'ils vivent chez leurs parents, et dans le village.

Par simple observation, les enfants enregistrent inconsciemment au fil des années des gestes, des séquences de chaines opératoires. C'est la période d'apprentissage qu’on pourrait appeler « passive ». Certains travaux leur sont cependant demandés très tôt: 
porter une charge, surveiller et transporter leur frère dernier-né (surtout pour les fillettes, mais pas exclusivement). On entraîne les enfants à porter des charges dès l'âge de quatre ou cinq ans ${ }^{6}$. Parfois, vers sept ou huit ans, on les emploie aux champs pour les mêmes travaux que les femmes.

Mais ce n'est que vers 10 ou 12 ans que les enfants commencent à s'exercer aux tâches de leur sexe: fabrication des tortillas, cuisine, broderie, lessive pour les filles; sarclage, conduite de mule, abattage des arbres pour les garçons. C'est la période d'apprentissage «active». Entre le début de cette phase et l'autonomie complète de l'individu, il ne s'écoule guère qu'un ou deux ans maximum.

Pendant toute l'enfance, filles et garçons observent l'ensemble des gestes et des séquences dans les techniques familiales; puis, à l'approche de l'adolescence, ils essaient de les reproduire en suivant le modèle indigène de la division sexuelle du travail. L'entourage n'intervient guère dans ce processus, sauf pour décider à partir de quel moment l'enfant est assez grand, assez fort, pour commencer à remplir telle ou telle tâche (phase « active »). Entre les adultes et les enfants, il n'y a pas pour ces travaux de relation caractérisée d'enseignant à enseigné, de maître à élève, de patron à apprenti. L'enfant apprend tout seul, et avec tous les gens, parents ou non, qui se trouvent là. Ce mode d'apprentissage est analogue à celui du langage.

Ainsi s'élabore et se transmet un lot de compétences communes aux deux sexes, indépendamment des performances techniques modelées par la vision indigène de la division sexuelle du travail. Tous les enfants à partir d'un certain d'âge savent comment accomplir certaines tâches, qu'elles soient ou non typiques de leur sexe. Lorsque les circonstances conduisent les adultes à effectuer des travaux habituellement réservés à l'autre sexe, il se peut bien sûr que les résultats soient moins bons. Les tortillas faites par des hommes seront moins fines peut-être. Mais ces différences seront toujours quantitatives, analogues aux variations d'habilité ou de rapidité entre les individus qu'on observe dans toutes les techniques. Elles ne seront pas qualitatives, c'est-à-dire opposant un savoir-faire à une ignorance, quel que soit le discours indigène à ce propos.

\section{Dans les savoir-faire particuliers}

Les techniques de spécialiste sont-elles plus « difficiles», et partant leur transmission exige-t-elle un maître? Ou encore sont-elles des secrets techniques qui ne sont transmis qu'à certaines personnes? On serait facilement enclins à lier difficulté, savoir-faire de spécialiste, et transmission par un maître. Pourtant, à Cuacuila, on trouve sans peine des exemples de techniques qui montrent que ces trois caractéristiques ne sont pas nécessairement liées. Bien plus, la notion de « difficulté » se révèle, à l'usage, extrêmement floue et liée à la subjectivité de l'observateur.

En réalité, on constate que lorsque les deux conditions de l'imprégnation sont remplies - techniques qui s'appuient sur le fonds commun culturel des gestes et expériences, et qui peuvent être observées fréquemment - un savoir-faire particulier emprunte aussi cette voie de transmission. Ce qui le maintient à l'état de savoir-faire particulier, c'est sa faible diffusion dans le village, sa faible base de reproduction. C'est le cas d'un certain nombre de techniques connues de quelques familles seulement.

Un exemple observé est celui de la charcuterie. Tous les Indiens du village sont bien loin de savoir tuer un porc, le dépecer, fabriquer la couenne grillée (chicharrón), le saucisson piquant (chorizo), le boudin, etc. Un nombre réduit de personnes connaissent 
les procédés, les chaînes opératoires. Parmi eux, un nombre encore plus réduit pratique effectivement l'abattage des porcs: ceux qui ont les capitaux nécessaires pour en faire une activité commerciale. Dans le village, qui compte 1500 habitants, le nombre des charcutiers pratiquant effectivement varie entre 4 et 6 chefs de famille.

Tous les travaux de charcuterie se font dans la maison et ses abords, avec l'aide de tous les membres de la maisonnée. Au sein des familles de charcutiers, les savoir-faire se transmettent par imprégnation. Les jeunes participent activement au travail du tri hebdomadaire de charcuterie dès l'adolescence. La division du travail dans la famille s'effectue non pas suivant des différences de compétences, mais par une simple division des tâches. Chacun a connaissance du processus complet.

Aussi, si les garçons ont les moyens financiers nécessaires, ils pourront s'établir à leur compte après leur mariage. Les filles sont aussi compétentes, mais pour qu'elles soient à la base d'une nouvelle unité de production après leur mariage, il faut qu'elles épousent un charcutier, ou que leur mari agriculteur apprenne spécialement le métier.

Pour celui qui n'est pas d'une famille de charcutier, l'imprégnation est en effet impossible. Il lui manquera l'observation répétée des chaînes opératoires, et la connaissance du processus intégral. Il devra, pour apprendre, s'adresser à un maître.

Ainsi, selon les conditions sociales de mise en œuvre, un même savoir-faire peut se transmettre par imprégnation, ou au contraire par un maître.

En l'occurrence, le caractère particulier d'un savoir-faire ne résulte pas de l'existence de secrets de fabrication institués. Cependant le jeu de la transmission strictement familiale jointe aux obstacles économiques (nécessité de capitaux) a des effets voisins. Elle limite à l'avance l'extension de certains savoir-faire, ralentissant ou empêchant leur généralisation. Des activités comme la boulangerie, la boucherie connaissent des situations analogues, du moins dans leurs grandes lignes.

L'existence de savoir-faire particuliers transmis par imprégnation met en évidence l'effet de différents niveaux de la réalité sociale sur les phénomènes techniques. On remarque en particulier que ce n'est pas toujours l'existence de difficultés d'ordre technique qui placent un savoir-faire dans les mains de spécialistes, mais des obstacles d'ordre social. Selon qu'il aura accès ou non à l'observation répétée de chaînes opératoires complètes, un individu accédera ou non à la compétence technique, pour les savoir-faire qui s'appuient sur des entraînements communs à tous les membres du groupe. Dans l'hypothèse où l'individu a pu acquérir la compétence nécessaire, il ne réalisera la performance que si certaines conditions extra-techniques sont remplies. Dans les exemples cités, la performance est liée à l'économie, et n'est possible que si l'on possède un capital monétaire à investir.

Voilà qui remet en cause des idées reçues sur la genèse ou le maintien des spécialisations techniques. Ce n'est pas toujours une croissance des exigences techniques qui conditionne la spécialisation, mais une exigence économique (un capital) qui conditionne la performance, laquelle conditionne la répétition, qui conditionne à son tour l'imprégnation.

\section{Comment s'effectue la transmission par un maître}

De même qu'il existe des savoir-faire de spécialistes qui se transmettent par imprégnation lorsque les conditions sont remplies, il existe aussi le cas de figure inverse, celui de savoirfaire généraux qui ne peuvent être transmis par cette seule voie. La large diffusion de certains savoir-faire dans le village ne suffit pas à assurer leur reproduction spontanée, et 
une relation de maître à apprenti est nécessaire. Sont dans ce cas les savoir-faire généraux masculins ou féminins, lorsqu'ils sont de véritables spécialisations, avec compétence exclusive d'un sexe, et non de simples divisions des tâches.

Puisqu'il n'y a pas de frontière rigide entre l'univers des hommes et celui des femmes, et que par conséquent l'observation des savoir-faire est possible à tous, qu'est-ce qui empêche la transmission par imprégnation dans les cas évoqués? Deux obstacles peuvent se présenter. Un seul d'entre eux est suffisant pour gêner la transmission par imprégnation.

Le premier obstacle possible déjà évoqué est le défaut de répétition de l'observation. Toutes les fois qu'elle sera insuffisante, le savoir-faire technique se transmettra mal tout seul. Le défaut de répétition se rencontre dans les techniques rarement mises en œuvre: par exemple, la construction d'une maison n'advient pas chaque année, mais seulement quatre ou cinq fois dans une existence. Comme c'est un travail d'homme, assisté d'autres hommes de la famille et d'un charpentier, les femmes n'accéderont pas à la compétence nécessaire. Le défaut de répétition a également un effet dans la transmission de toutes les techniques, même fréquentes, que certaines personnes ont rarement l'occasion d'observer. Ainsi en agriculture, les femmes assistent rarement au labour, et sont incompétentes pour cette tâche particulière ${ }^{7}$. Il en est de même pour l'abattage des arbres, la chasse, etc. Inversement, les hommes n'assistent pas à certaines préparations culinaires qui se font quand ils sont aux champs, à la lessive qui se fait au bord de la rivière et non dans l'espace domestique, etc. Ce ne sont là que quelques exemples où l'imprégnation est impossible, faute de répétition.

Le second obstacle à la transmission par imprégnation est la nécessité pour certains savoir-faire, d'entrainements corporels et intellectuels spéciaux qui ne sont pas transmis en même temps que le fonds commun culturel de la population. On va détailler ici le cas de deux techniques, l'une qui est un savoir-faire général féminin, l'autre qui est un savoir-faire particulier féminin, pour montrer quels entraînements spéciaux peuvent être nécessaires.

\section{La transmission par un maître d’un savoir-faire général féminin: la broderie}

La broderie est une technique connue de la majorité des femmes de Cuacuila. Les hommes du village sont incapables de la pratiquer, bien qu'ils aient très souvent l'occasion de voir des femmes broder.

\section{Comment les fillettes apprennent à broder}

La technique est transmise de mère à fille, ou de sœur aînée à sœur cadette. La méthode de transmission employée vaut pour d'autres techniques présentant la nécessité d'un maître. Peu avant l'adolescence, vers 11 ans, les fillettes s'essaient à broder. Elles commencent par des travaux peu ambitieux: des broderies de petite taille, sur des serviettes par exemple. Ce n'est que plus tard qu'elles s'attaqueront aux broderies plus grandes des blouses de femmes. D'abord, la mère ou la sœur aînées montrent à la fillette comment on fait. Puis la fillette essaie de reproduire les gestes et les résultats qu'elle a vus. De temps à autre, la mère ou la sœur regarde ce que produit la petite. Elles critiquent ou approuvent d'un geste, d'un mot, d'un rire, d'une plaisanterie, mais jamais par de longues phrases. Elles corrigent très rarement elles-mêmes le résultat. Pas de surveillance constante non plus: entre les interventions de l'adulte faisant office de maître, la fillette affronte comme elle peut les résistances de la matière. Peu importe le temps qu'elle met à parvenir à un 
résultat proche de celui qui est désiré. Un premier travail de broderie, très simple et très court, peut mettre des semaines avant d'être terminé. La fillette acquiert ainsi, sans phrases inutiles, l'entraînement physique et intellectuel la rendent capable de broder. Peu à peu le savoir-faire «s'incorpore».

Mais quels entrainements spéciaux sont nécessaires pour cette technique? On ne peut les découvrir en partie qu'en considérant de près les chaînes opératoires, ou, tout le moins, certaines caractéristiques du rapport à la matière.

\section{Les caractéristiques de la technique de broderie}

La broderie à l'aiguille pratiquée aujourd'hui à Cuacuila est probablement une technique venue d'Europe. On l'exécute sur des toiles de coton blanc, à l'aide d'aiguilles et de fils de coton de couleur. Tous ces matériaux et outils sont de fabrication industrielle et achetés dans les boutiques de la ville. Les motifs brodés sont très souvent aussi d'origine non amérindienne. Ils s'inspirent de l'imagerie religieuse catholique, ou des symboles nationaux mexicains, ou encore de modèles d'« ouvrages de dames » vendus dans le commerce. Certains motifs apparaissent plus « traditionnels». En réalité, ils sont souvent des motifs européens anciens. Il existe également quelques motifs d'origine nettement précolombienne.

Malgré tout, les broderies indiennes actuelles ont un style particulier incontestable. La manière de traiter et d'agencer les motifs, et aussi la technique d'exécution choisie sont les composantes de ce style. On considérera ici principalement les effets et les exigences de la technique d'exécution.

Quatre points de broderie sont connus à Cuacuila: le point de croix; le point de chausson (ou de zigzag); le point de feston; le point lancé. Le point de croix est rarement utilisé, et seulement pour border des serviettes. Les points de chausson et de feston servent à faire des coutures et des bordures. Le point lancé est le plus fréquent. C'est lui qu'on va étudier plus particulièrement.

À Cuacuila, la broderie au point lancé est effectuée de façon très particulière: le fil de couleur est passé sur le tissu parallèlement à la trame, et jamais dans le sens de la chaîne ou dans des positions obliques. Il résulte d'une marque culturelle. La broderie au pont lancé s'apparente au tissage et elle reproduit à l'aiguille un effet de broché. Les motifs apparaissent en négatif à l'envers du tissu. Leurs contours prennent des formes géométriques, comme s'ils étaient tissés.

Pour exécuter correctement la broderie, l'aiguille doit être piquée à un endroit très précis dans le tissu: le fil de couleur doit être tendu de façon parfaitement parallèle aux fils de trame, et sa partie visible doit avoir la longueur exacte requise par les contours du motif. Ces exigences sont celles que l'on connaît pour les broderies sur canevas. En cas d'erreur non rectifiée immédiatement, c'est toute la composition qui risque d'être déformée. Cette technique de broderie suppose que la brodeuse compte les fils de chaîne avant de piquer l'aiguille.

La toile servant de support à la broderie est utilisée comme un plan finement quadrillé (par exemple un papier millimétré) où l'on tracerait des lignes horizontales de différentes longueurs pour former des figures géométriques. Sans même parler du canevas bien connu chez nous, le traçage des motifs s'apparente à ces techniques d'impression de dessins effectuées parfois par des ordinateurs. On peut dire que, dans l'esprit de la brodeuse, tout se passe comme si la structure quadrillée du tissu s'imposait aux motifs. Or ce n'est nullement nécessaire en broderie: on peut au contraire traiter 
le tissu comme un papier uni sur lequel on est libre de dessiner ou de peindre tout ce qu'on veut, fut-ce à l'aide de fils et d'aiguilles.

Les entraînements spéciaux nécessaires

La technique employée requiert des entraînements spéciaux. Les uns sont communs à tous les travaux de broderie à l'aiguille. D'autres découlent des exigences spécifiques du style géométrique des motifs. Parmi les capacités communes à toutes les sortes de broderie, il faut citer: une bonne coordination entre la main et la vue, pour piquer l'aiguille à l'endroit désiré; une acuité visuelle d'autant meilleure que le support est plus fin et les motifs plus petits; une bonne perception des formes pour donner des contours nets aux motifs; une bonne perception des rapports entre les formes, pour centrer les motifs et réussir la composition d'ensemble. Aucune de ces capacités n'est normalement développée par des activités comme l'agriculture, les tâches d'entretien ménager et autres activités quotidiennes.

Parmi les compétences particulières requises par la technique du point lancé telle qu'elle est pratiquée à Cuacuila, on trouve: une acuité visuelle telle que la brodeuse puisse distinguer chacun des fils de chaîne du tissu, et chacun des fils de trame; une capacité d'attention permettant à la tisseuse d'identifier dans toute la largeur de l'ouvrage le trajet d'une même suite, pour garder l'horizontale; des techniques de comptage pour se donner des points de repère et les utiliser correctement. En outre pour certaines variétés de blouses brodées, tous les agencements de motifs et les comptages de fils doivent être
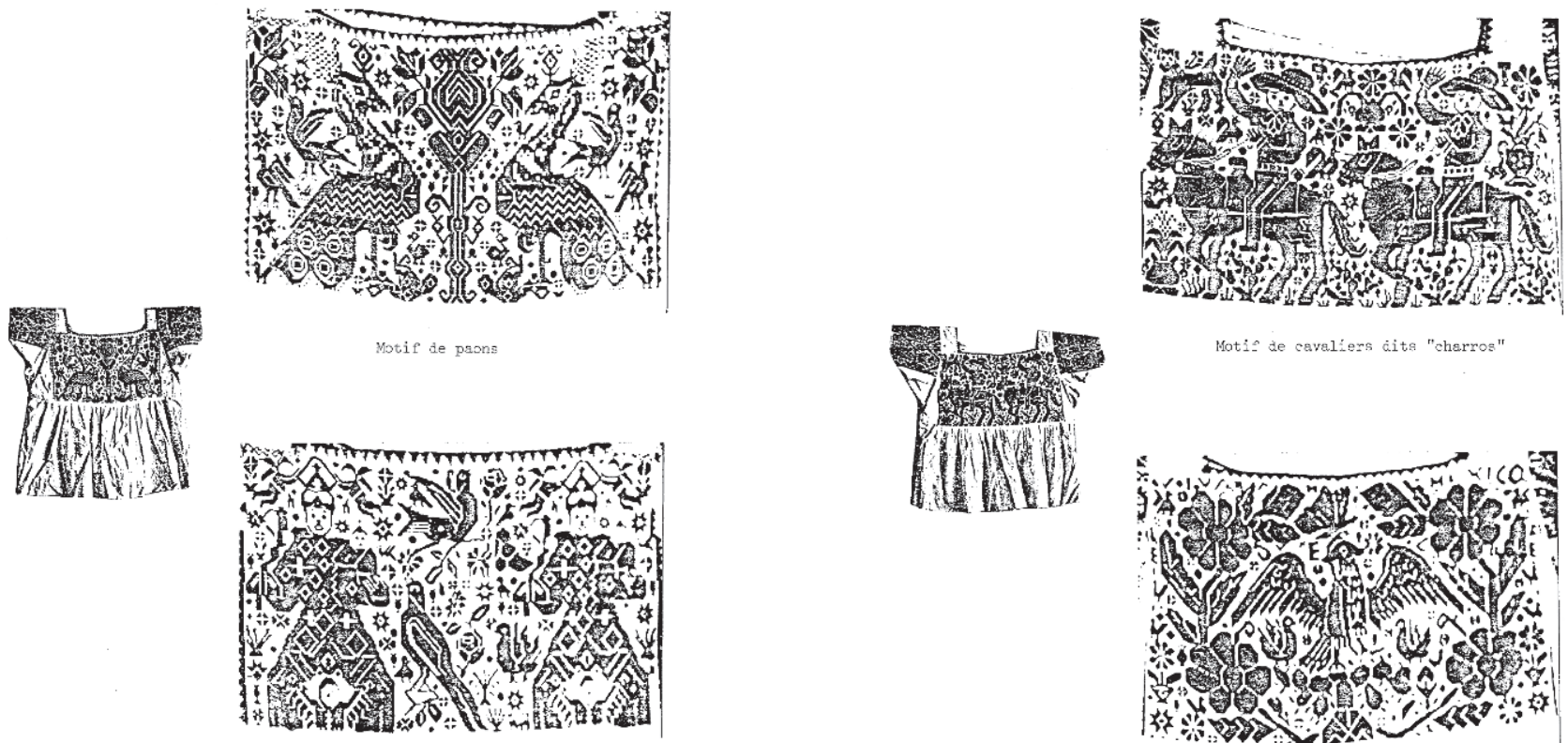

Motif de "Saint Antoine de Padove"

Figure 2

Broderies de blouses de femmes,

Cuacuila (Huauchinango, Puebla) : motif de cavaliers dits « charros » et motif d'aigle, symbole officiel de Mexico.

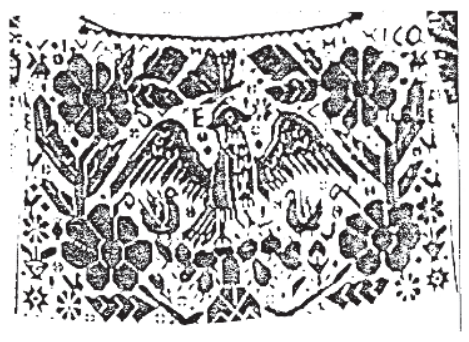

Motif d'aimle, symbole of siciel de Maxico

Cuacuila (Huauchinango, Puebla) : motif

de paons et motif de « Saint Antoine de Padoue ».
Figure 1

Broderies de blouses de femmes, 


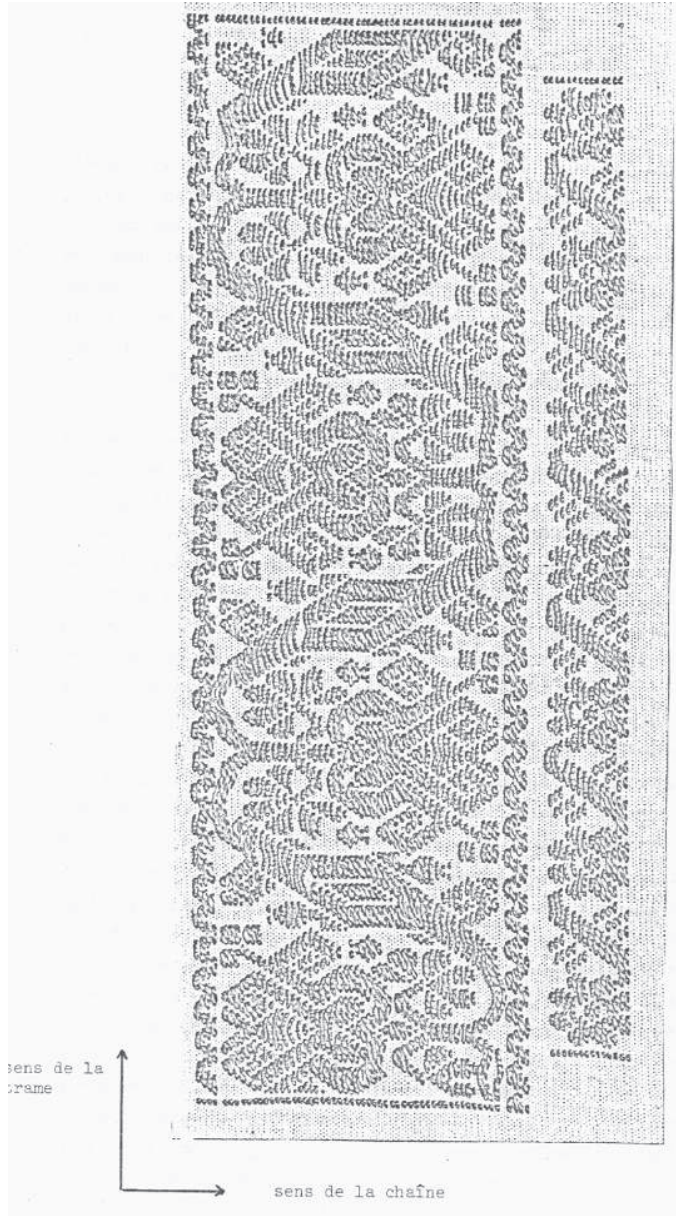

\section{Figure 3}

Broderie sur toile fine, grandeur nature, Huilacapixtla (Huauchinango, Puebla) : sens de la trame et sens de la chaîne. programmés dès le premier passage de l'aiguille, la broderie étant produite rang horizontal par rang horizontal, et non motif par motif (voir figure 3).

Si on réalise bien les exigences d'ensemble de cette technique, on se rend compte à quel point ce type de travail demande, outre de bons yeux, une part considérable d'activité et d'entraînement mental abstrait. Cette part intellectuelle est d'autant plus grande que les brodeuses ne dessinent jamais à l'avance sur le tissu les contours des motifs à broder, contrairement à ce qui se fait dans d'autres groupes humains, par exemple en France. Elles brodent sur le tissu, sans aucune marque concrétisée: le dessin et les repères sont présents dans leur tête et non sur l'ouvrage. Elles utilisent cependant des modèles et ne font pas toute la broderie de mémoire: ce sont de vieilles blouses ou des bouts de tissu sur lesquels sont déjà brodés les motifs qu'elles veulent reproduire. $\mathrm{Au}$ cours de leur travail, elles consultent de temps en temps le modèle, qu'elles nomment copia ou copina.

Toutes les femmes sont capables de broder sans dessin préalable. Pour quelqu'un de non entrainé, c'est impossible d'emblée ${ }^{8}$. Ci-après:

Est-ce à dire que le dessin est une technique inconnue à Cuacuila? ou alors que son application à la broderie n'a pas été encore découverte? Il n'en est rien. Certaines femmes sont capables de dessiner, et aussi d'utiliser des dessins faits sur le tissu à broder. Mais ces femmes ne se servent des possibilités des crayons, du papier-calque et du papier carbone que dans un seul cas: celui de l'invention d'un nouveau modèle de broderie. Elles recopient ou décalquent une image quelconque (image pieuse ou calendrier publicitaire) qui constituera le motif central de la broderie. Puis elles la reportent avec du papier carbone sur le tissu à broder. Elles brodent alors directement, en transcrivant en formes géométriques typiques les contours dessinés, et en complétant au fur et à mesure la composition d'ensemble par des motifs annexes, lesquels ne sont pas toujours dessinés à l'avance. Les motifs inventés sont ensuite recopiés d'après la blouse brodée par les autres femmes du village.

La broderie est un savoir-faire général féminin, à Cuacuila, mais l'invention des motifs est un savoir-faire particulier.

On voit qu'un savoir-faire général peut aussi être transmis par un maître, contrairement à l'idée qu'on pouvait avoir a priori.

\section{La transmission par un maître d'un savoir-faire particulier : le tissage}

Le dernier cas de figure ne pose pas de problème quant à sa possibilité concrète: on conçoit facilement comment un savoir-faire technique peut être transmis par un maître et rester particulier.

Mais la grille d'analyse proposée reste utile pour étudier les places tenues dans le groupe villageois par les différents savoir-faire de ce type. Pour illustrer cette perspective, on développera le cas du tissage, en parallèle avec celui de la broderie. 
Actuellement à Cuacuila, la broderie est un savoir-faire général féminin, et le tissage un savoir-faire particulier féminin. Mais ces deux techniques n'ont pas toujours tenu ces mêmes places dans le village.

La broderie est, rappelons-le, une technique d'origine européenne acquise par les Indiennes. Il semble qu'elle ait été introduite dans les villages au cours du xix siècle, et que, vers sa fin, elle se soit généralisée. À Cuacuila, la broderie se maintient bien aujourd'hui, tout en ayant connu des évolutions dans les procédés (changements de points), dans les matériaux, dans les motifs.

Le tissage est au contraire effectué suivant une ancienne technique amérindienne, qui était, dans les périodes antérieures, connue et pratiquée par la plupart des femmes. Aujourd'hui, ce savoir-faire se raréfie: très nombreuses sont les femmes qui, non seulement ne tissent plus, mais ont perdu la compétence nécessaire. Le procédé lui-même a connu très peu d'évolutions internes, les changements consistant surtout à utiliser des matériaux industriels, et à introduire quelques nouveaux motifs. La technique elle-même (outils, gestes armures) est restée la même, à très peu de chose près. Ce qui a changé, c'est la place du tissage dans la répartition des savoir-faire féminins: de général, il est devenu particulier.

On peut schématiser, dans l'état actuel des connaissances, l'évolution des places des deux savoir-faire techniques de la façon suivante:

\begin{tabular}{|l|c|c|}
\hline \multicolumn{1}{|c|}{ SAVOIR-FAIRE } & BRODERIE & TISSAGE \\
\hline PÉRIODE & & \\
\hline Période la plus ancienne & Savoir-faire absent (européen) & Savoir-faire général féminin \\
\hline Fin du xixe siècle & Savoir-faire général féminin & Savoir-faire général féminin \\
\hline Actuellement (1970) & Savoir-faire général féminin & Savoir-faire particulier féminin \\
\hline
\end{tabular}

La généralisation de la technique de broderie correspond à l'introduction, vers la fin du siècle dernier, d'un nouvel élément du vêtement féminin, les blouses de femmes. Les blouses ne se sont pas substituées à un autre vêtement, mais ajoutées au costume féminin de la région qui se perpétuait depuis les temps précolombiens. Dans le cas de la broderie, c'est l'adoption d'un nouveau produit qui a servi de support à l'acquisition d'un nouveau savoir-faire technique.

Mais les changements dans l'extension des savoir-faire du vêtement ne suivent pas toujours les changements dans les produits utilisés. Ainsi le tissage a pu reculer sans que l'usage de produits tissés régresse dans les mêmes proportions. Les femmes de Cuacuila portent toutes encore aujourd'hui les trois éléments du costume précolombien: la jupe, la ceinture, et le quechquemitl, petit vêtement passé autour du cou et couvrant plus ou moins bien le buste. Ces éléments sont toujours tissés selon les techniques amérindiennes. Mais comme la production villageoise actuelle est insuffisante, les femmes achètent ces vêtements indigènes aux tisseuses de villages voisins.

Une question se pose alors. Puisque les besoins en produits tissés restaient les mêmes, pourquoi le savoir-faire de tissage a-t-il régressé à Cuacuila? Est-ce en raison de difficultés particulières dans la transmission des entraînements spéciaux? Faut-il chercher le sens de ce changement sur un plan purement technique, ou faut-il le rechercher dans d'autres niveaux de la réalité sociale?

Les femmes de Cuacuila donnent spontanément des raisons d'ordre technique à leur désaffection vis-à-vis de cette technique. Le tissage, disent-elles, est trop trabajoso, mot 
espagnol qui signifie pour elles à la fois fatigant et difficile. Elles ajoutent aussi que le tissage demande beaucoup trop de temps. Que recouvrent exactement ces arguments?

On a montré comment les procédés et les pratiques techniques de la broderie actuelle demandaient des entraînements spéciaux, pas faciles du tout à acquérir. Peut-on dire que, pour les Indiennes, le tissage est vraiment plus « difficile »? C'est possible, bien qu'à première vue, on puisse aussi supposer que beaucoup d'exigences du tissage sont voisines de celles de la broderie (coordination entre les mains et les yeux, acuité visuelle, méthodes de calculs, mémoire des motifs, etc.).

Cependant il est manifeste que le tissage demande des entraînements supplémentaires pour deux raisons techniques. En premier lieu, contrairement à la broderie, c'est le corps tout entier de la tisseuse qui est actif dans le procédé amérindien et pas seulement ses mains. Le métier à tisser ne comporte pas de bâti, mais deux bâtons faisant fonction d'ensouple et de poitrinière, sur lesquels la chaîne est fixée. L'ensouple est accrochée par un cordon à un point fixe quelconque situé environ à un mètre du sol. La poitrinière est fixée par une ceinture de palme aux reins de la tisseuse qui est agenouillée ou assise sur le sol. C'est donc le corps même de la tisseuse qui assure la tension des fils de chaîne et les variations de tension éventuellement nécessaires. Cette posture peut évidemment à la longue entraîner un type de fatigue absent de la broderie.

De la même manière, il est possible d'observer en quoi le tissage peut apparaître effectivement comme une technique plus « difficile ». On trouve en effet dans la chaîne
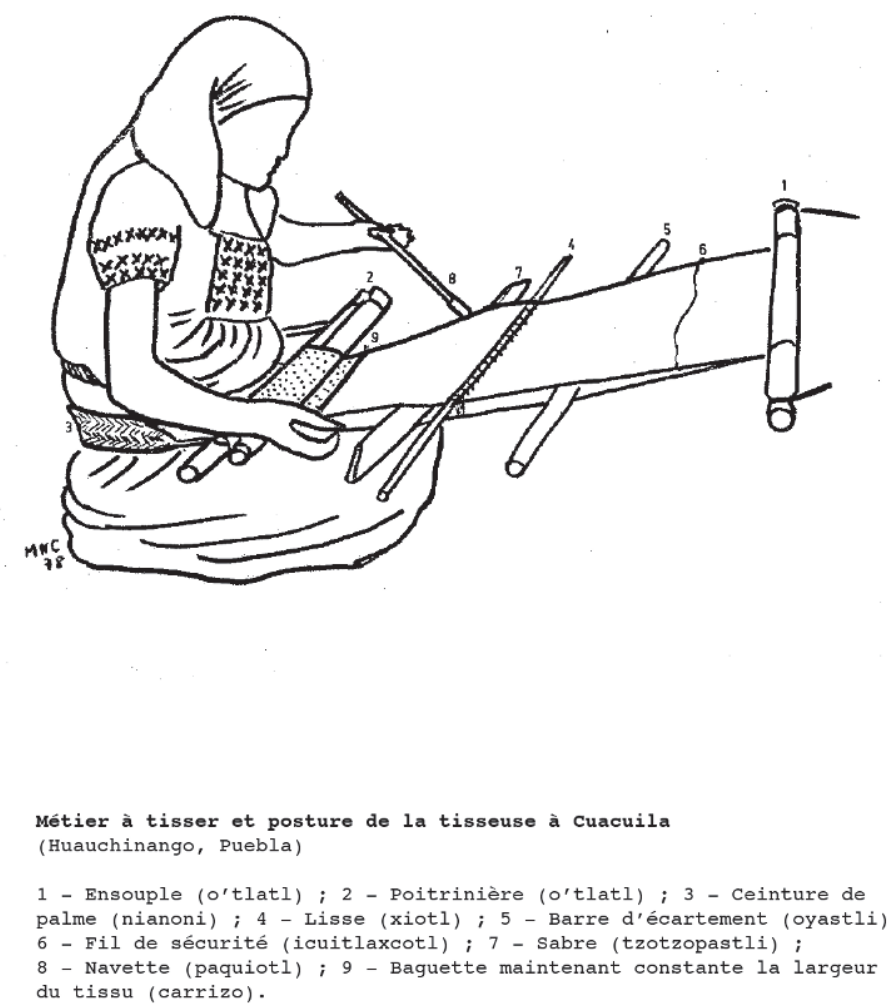
opératoire une opération très délicate de préparation, l'ourdissage, qui est absent de la broderie. De l'avis même des tisseuses, l'ourdissage demande une grande concentration intellectuelle. D’après une étude récente effectuée dans une région voisine, « les femmes n'aiment pas être dérangées » pendant qu'elles ourdissent ${ }^{9}$. On sait que les entraînements particuliers sont requis par cette opération, notamment que les Indiennes ont élaboré des systèmes de comptage des fils pour programmer les armures. Le tissage semble donc bien demander des entraînements spéciaux supplémentaires.

Outre la fatigue et la difficulté, les Indiennes invoquent la lenteur de la production comme raison de leur désaffection. De fait c'est là une caractéristique évidente, aisément vérifiable et mesurable par un observateur extérieur.

On pourrait s'en tenir là et admettre que l'ensemble des raisons invoquées peut expliquer le passage du tissage de la situation de savoir-faire général féminin à celle de savoirfaire particulier féminin. Les difficultés et les inconvénients inhérents à la technique amérindienne auraient rebuté un certain nombre de femmes; en conséquence celles-ci auraient renoncé à acquérir ce savoir-faire. Mais une telle interprétation laisse bien des questions sans réponses: le tissage n'était-il pas aussi fatigant, difficile et lent pour les grands-mères et arrière-grand-mères des Cuacuiltèques? Celles-ci pourtant le pratiquaient largement, et pas nécessairement sous la contrainte matérielle. Des étoffes industrielles assez bon marché et substituables aux tissus locaux se trouvaient déjà à leur portée et l'abandon du tissage était matériellement possible. De même on peut se demander pourquoi dans certains villages voisins la difficulté et la lenteur du procédé n'ont pas conduit à une désaffection aussi prononcée pour la même technique. Bref puisque le procédé lui-même n'a pas changé l'attitude actuelle des Cuacuiltèques ne peut s'expliquer par les seules raisons techniques invoquées.

Dès lors les questions posées par le recul de ce savoir-faire obligent à se tourner vers d'autres niveaux de la réalité sociale. Elles conduisent à considérer non plus seulement les caractéristiques du procédé particulier mais l'ensemble des activités féminines. Une seule explication semble possible. L'extension d'un savoir-faire comme le tissage ne serait conditionnée ni par les difficultés particulières du procédé, ni par son mode de transmission. Elle serait conditionnée par la place occupée à un moment donné parmi les autres activités féminines. De fait à Cuacuila le tissage entre en concurrence avec d'autres occupations jugées plus « utiles » par les villageoises. C'est pour cette raison qu'il apparaît aujourd'hui comme plus fatigant, plus difficile et moins productif.

Sans entrer dans les détails, on peut signaler que le recul du tissage est allé de pair avec la spécialisation du village dans la production de légumes pour le marché national. Avec l'intensification du travail agricole qu'a connu Cuacuila depuis les années trente, beaucoup de familles préfèrent que les femmes consacrent plus de temps aux tâches servant de près ou de loin la production de légumes et leur commercialisation. Ce nouveau temps de travail féminin n’a pas été pris seulement sur le repos mais aussi sur celui consacré à certaines productions d'auto-consommation, en premier lieu au tissage. 
Dans les villages voisins qui n'ont pas connu le même développement en agriculture, le tissage s'est maintenu comme production complémentaire dans les familles d'agriculteurs. Les tisseuses de ces villages bénéficient ainsi du petit marché local qui s'est développé à la suite du recul du tissage d'auto-consommation dans d'autres localités, telle Cuacuila. Le changement d'extension du savoir-faire du tissage serait donc une conséquence de processus plus généraux de division du travail entre les villages indigènes, effet du développement du marché national.

L'étude qui a été menée ici à partir du cas concret d'un village indien n'a abordé que deux aspects parmi d'autres des pratiques techniques, ceux de l'extension des savoir-faire incorporés et des variations de leur mode de transmission. Bien d'autres aspects seraient à analyser. Mais l'expérience tentée montre déjà à quel point l'étude des pratiques techniques est essentielle. À travers la prise en considération des savoir-faire, c'est l'inscription des techniques dans l'histoire des groupes humains qui apparaît et non plus seulement l'histoire des procédés techniques. 
1. J'ai repris dans ce paragraphe des constatations faites collectivement par l'équipe Techniques et Culture.

2. Yves Barel, « La Ville avant la planification urbaine ». In Prendre la ville, Paris : Anthropos, 1977 : 16-19.

3. Organisation Scientifique du Travail. Nom donné aux disciplines qui, suivant les principes de F. W. Taylor, tentent de rationaliser les procès de travail dans les entreprises industrielles pour accroître la productivité (ergonomie, organisation et méthodes, etc.).

4. Le mot performance n'a pas ici sa connotation, habituelle en français, de mesure de capacité de l'individu.

5. La distinction entre hommes et femmes à laquelle on se réfère est bien entendu celle opérée par la population indigène, et non la distinction biologique.

6. Il est intéressant de remarquer que cette incorporation générale du savoir-faire de portage était déjà pratiquée du temps des Aztèques. Personne, pas même l'empereur, ne devait jamais se déplacer sans porter quelque chose, fut-ce des cailloux.

7. Ceci apporte une nuance à l'idée d'une compétence générale des femmes en agriculture, mais ne la remet

\section{NOTES ET RÉF́ERENCES DE L'ITTRODUCTION}

1. Image d'ouverture : tissage dit en courbe d'un couvrebuste en laine de mouton écrue, à galon tissé rouge, Cuacuila, Huauchinango, Puebla.

2. Comme je le sus plus tard, une préoccupation semblable guidait alors les recherches en sociologie des sciences : cesser d'opposer «l'humain » au « nonhumain » (voir les travaux de Callon et Latour à partir de 1981).

3. Cette partie d'histoire de la sociologie du travail est retracée par Stroobants, M. 1993, Savoir-faire pas en cause. En effet le labour est une opération facultative. Certains paysans, surtout les plus pauvres, se contentent de préparer la terre à la houe, outil que les femmes savent manier.

8. J'en ai fait personnellement l'expérience: j'ai tenté de copier une broderie sans dessin préalable des contours des motifs. Sur un papier millimétré représentant la toile, j'ai tracé des lignes horizontales représentant les fils de couleur, en commençant de la même façon que la brodeuse. Le résultat a été catastrophique. La première erreur de comptage non rectifiée s'est répercutée sur l'ensemble du dessin, déformant complètement les motifs. Je n'avais pas su me donner des points de repère efficaces. Cette tentative m'avait pourtant pris beaucoup de temps et occasionné une fatigue visuelle et intellectuelle assez considérable.

9. Kandt, Vera B., Arts and crafts of Zacatipan, a Nahuat village in the Sierra de Puebla, State University of Leiden: Pays-Bas, 112 p. mimeo. et compétence au travail, Université de Bruxelles et Stroobants, M. 2009, «Dénouer les ficelles du métier », Techniques \& culture 51 : 164-179.

4. Rapport de l'équipe de recherche 191 du CNRS Techniques et Culture, 1986, miméo.

5. Chamoux, M.-N. 1981 Les savoir-faire techniques et leur appropriation: le cas des Nahuas du Mexique, L'Homme, XXI (3) : 71-94.

6. Delbos, G. \& Jorion, P. 1984 La transmission des savoirs. Paris: Maison des Sciences de l'Homme. 